Check for updates

Cite this: RSC Adv., 2019, 9, 15976

Received 10th January 2019

Accepted 30th April 2019

DOI: 10.1039/c9ra00243j

rsc.li/rsc-advances

\section{Microwave-assisted synthesis of glutathione- coated hollow zinc oxide for the removal of heavy metal ions from aqueous systems $\uparrow$}

\author{
Lateef Ahmad Malik, Arshid Bashir, Taniya Manzoor and Altaf Hussain Pandith (iD *
}

\section{Introduction}

Water is indispensable to living creatures on earth; its contamination by toxic heavy metals, such as $\mathrm{Hg}^{2+}, \mathrm{Pb}^{2+}$, and $\mathrm{Cd}^{2+}$, is a challenging issue. Among these ions, $\mathrm{Hg}^{2+}$ is considered the most dangerous metal ion due to its tendency for biomagnification. Some potent sources of mercury pollution include chloralkali wastewater, oil refineries, power generation plants, paper and pulp manufacturing, rubber processing, and fertilizers industries. ${ }^{1}$ From the toxicity point of view, there are three main forms of mercury: inorganic mercury salts, organic mercury compounds and metallic mercury. ${ }^{2}$ Inorganic mercury salts, which are water soluble, can cause gut irritation and

Department of Chemistry, University of Kashmir, Hazratbal, Srinagar-190006, Kashmir, India. E-mail: altafpandit23@gmail.com; Fax: +91-194-2414049; Tel: +91194-2424900; +91-990-6424293

$\dagger$ Electronic supplementary information (ESI) available: Fig. S1: DLS studies of the particle size distribution by intensity: (a) variation of the average hydrodynamic diameter of Glu@h-ZnO particles with pH and (b) the average particle size of $\mathrm{h}-\mathrm{ZnO}$ at a constant $\mathrm{pH}$ value of 7 . Fig. S2: plot of relative frequency (\%) as a function of $\zeta$ at various $\mathrm{pH}$ values for Glu@h-ZnO (a) and for h-ZnO at $\mathrm{pH} 7$ (b). Fig. S3: percentage removal of $\mathrm{Hg}^{2+}, \mathrm{Cd}^{2+}$ and $\mathrm{Pb}^{2+}$ ions by Glu@h-ZnO at $\mathrm{pH}=7, T=25^{\circ} \mathrm{C}$. Fig. S4: variation of zero point energy as a function of bond length validating the preferable selectivity of metal ion binding with glutathione: (a) $\mathrm{Pb}^{2+}$; (b) $\mathrm{Cd}^{2+}$; and (c) $\mathrm{Hg}^{2+}$. See DOI: 10.1039/c9ra00243j severe kidney damage. Organic mercury compounds, which are fat soluble, can cross the blood-brain barrier and cause neurological damage. Mercury metal in vapour form can reach the brain and leads to tremors, depression, and behavioural disturbances. Furthermore, metallic mercury may get biotransformed into organic mercury by some bacteria and after entering the food chain it could cause a Minamata-like tragedy. ${ }^{3}$ Cadmium and lead also have serious toxic effects on living beings. Excessive amounts of cadmium cause kidney damage, renal dysfunction, anaemia, hypertension, etc. ${ }^{\mathbf{4}}$ while lead poisoning seriously affects the nervous, renal and cardiovascular systems. ${ }^{5}$ It is now very important to lower the concentrations of these toxic metal ions to trace levels as they do not have any propensity to be degraded or destroyed. ${ }^{6}$ This apprehension has led to stringent regulations regarding acceptable metal concentrations in water. ${ }^{7}$ There are many methods that can be employed for the removal of these toxic metal ions. Photo-catalytic reduction of some toxic forms of metal ions, like hexavalent chromium, to a less toxic form is a well-known method reported for waste water treatment. ${ }^{\mathbf{8} 9}$ Conventional methods, including chemical precipitation, membrane separation, ion exchange, and evaporation, are not desirable because of their low capacities and low removal rates for metals other than $\mathrm{Hg}^{2+}$. Precipitation employing sulfide ions cannot reduce the concentrations of heavy metals below acceptable drinking 
levels. ${ }^{10}$ The adsorption method is quite attractive because of its simple design, low cost, swift operability, and high removal efficiency. ${ }^{11}$ Various materials, such as zeolites, ${ }^{12}$ metal phosphates,${ }^{13-15}$ polymers,${ }^{16}$ biomaterials,${ }^{17}$ layered double hydroxide $(\mathrm{LDH})$-based materials ${ }^{18}$ and sorption resins, ${ }^{19}$ have been employed for the adsorption of metal ions. However, nanostructured metal oxides are quite useful in this field as they usually have a large surface area and better removal efficiencies, which make the treatment more economical. ${ }^{20} \mathrm{ZnO}$ nanoparticles have been employed in this field because of their great biocompatibility, high adsorption capacity, high specific surface area, recyclability, reusability, surface modifiability, and low cost. ${ }^{21-24}$ However, the use of nanoparticles in wastewater treatment may cause harmful effects when exposed to the environment owing to flaws in the recovery and disposal issues. ${ }^{25}$ Therefore, mechanical supports are used to hold the nanoparticles for possible applications in wastewater treatment. Use of surface modifying agents is an effective way of removing metal ions from water. ${ }^{26,27}$ Glutathione, which is a tripeptide, is well-known for its chelation with metal ions in biological systems, which is central to natural detoxification of heavy metals via the formation of complexes. ${ }^{28}$ Hollow and porous structures ${ }^{29-32}$ possess a high specific surface area and, as a result, they find applications in many fields. Such structures possess several different areas of contact, like borders and inner and outer surfaces, which is quite useful for enhanced adsorption properties. The method of synthesis, reaction time and many other factors play a crucial role in determining the size, morphology and composition of the final product. ${ }^{33}$ Using MW conditions, reactions can be accelerated and selectivity can be obtained by choosing appropriate MW parameters. It offers advantages like instantaneous and rapid heating, hightemperature homogeneity, selective heating and, in addition, the synthesis and morphology of nanomaterials can be modified in a better way by the efficient and controlled heating provided by MW irradiation. ${ }^{34}$

Here, we report the synthesis of glutathione-coated hollow zinc oxide (Glu@h-ZnO) under microwave conditions using polystyrene as the template. The as-synthesized composite material, after being characterized by various techniques, was used as an adsorbent for the removal of $\mathrm{Cd}^{2+}, \mathrm{Pb}^{2+}$ and $\mathrm{Hg}^{2+}$ ions from an aqueous system. The material was found to possess the highest affinity towards the uptake of $\mathrm{Hg}^{2+}$ ion so further adsorption studies, including adsorption isotherms and kinetics, were carried out for this particular ion only. The adsorption of metal ions by Glu@h-ZnO was also tested by theoretical calculations using DFT and good agreement was obtained between DFT and experimental results.

\section{Experimental section}

\subsection{Materials}

Styrene $\left(\mathrm{C}_{8} \mathrm{H}_{8}\right)$ as the building block of polystyrene (PS), polyvinyl pyrrolidone k-30 (PVP) as a stabilizing agent and sodium persulfate $\left(\mathrm{Na}_{2} \mathrm{~S}_{2} \mathrm{O}_{8}\right)$ as a radical initiator were purchased from Merck and were used without any further purification or processing. Zinc acetate as a source of zinc and reduced glutathione $\left(\mathrm{C}_{10} \mathrm{H}_{17} \mathrm{~N}_{3} \mathrm{O}_{6} \mathrm{~S}\right)$ were purchased from Sigma-Aldrich. In addition, lead nitrate, cadmium nitrate and mercuric chloride, all purchased from Merck, were used to carry out the adsorption studies. Ammonium hydroxide was used for precipitation of zinc ions while ethanol and water were used as the reaction medium.

\subsection{Synthesis}

2.2.1 Synthesis of polystyrene (PS). Polystyrene was synthesized from styrene by dispersion polymerization method, as already reported in the literature..$^{35}$ In this typical synthesis, styrene and PVP in a ratio of $2: 1(\mathrm{w} / \mathrm{w})$ were mixed in ethanol under continuous stirring at $343 \mathrm{~K}$. A few drops of sodium persulfate solution were added to polymerize the styrene and the solution was continuously stirred for the next $4 \mathrm{~h}$. The mixture was then centrifuged, washed with ethanol and deionised water many times in order to remove PVP and other impurities, and then dried at $300 \mathrm{~K}$ in an oven.

2.2.2 Synthesis of hollow zinc oxide nanoparticles (h-ZnO). To synthesize the hollow zinc oxide nanoparticles, PS was used as the template and an ethanol-water system was used as the reaction medium. An aqueous solution of $10^{-3} \mathrm{M}$ zinc acetate dihydrate and an ethanolic dispersion of PS were mixed together and the mixture was sonicated for 1 hour in an ultrasonic bath, followed by continuous stirring on a magnetic stirrer for the next 2 hours. A few drops of ammonium hydroxide were added as the precipitating agent and the mixture was irradiated by microwaves in a microwave synthesizer (Anton Paar monowave 100) at a temperature of $393 \mathrm{~K}$ for 10 minutes. Polystyrene coated with zinc oxide so obtained was centrifuged, washed with ethanol and water, and then calcined in a muffle furnace up to $800 \mathrm{~K}$. During this heating, PS decomposes ${ }^{36}$ to $\mathrm{CO}_{2}$ and $\mathrm{H}_{2} \mathrm{O}$, leaving behind hollow zinc oxide particles.

2.2.3 Synthesis of glutathione-coated hollow zinc oxide (Glu@h-ZnO). Calcination of $\mathrm{ZnO}$ at a high temperature eliminates hydroxyl groups that are present on the surface of the $\mathrm{ZnO}$ particles, ${ }^{37}$ so the calcined $\mathrm{ZnO}$ was washed with deionised water to regenerate the surface-bound $-\mathrm{OH}$. Reduced glutathione and h-ZnO particles in a ratio of $1: 1(\mathrm{w} / \mathrm{w})$ were mixed together in a limited amount of methanol, as reported in the literature. ${ }^{38}$ The mixture was sonicated for more than 1 hour and stirred on magnetic stirrer to ensure complete interaction between the hydroxyl groups of $\mathrm{h}-\mathrm{ZnO}$ and glutathione to form Glu@h-ZnO. The whole synthetic procedure for Glu@h-ZnO is shown in Scheme 1.

\subsection{Characterization}

Fourier transform infrared (FTIR) spectra of the samples were recorded over the range of $400-4000 \mathrm{~cm}^{-1}$ using a Bruker (Alpha 200486) FTIR spectrometer. The crystalline structure of the prepared samples was characterized by X-ray diffractometer (Ultima-IV, Rigaku Corporation, Tokyo, Japan) using $\mathrm{Cu} \mathrm{K} \alpha$ radiation. The microscopic analysis was carried out using a scanning electron microscope (Hitachi, S3000H. Japan) and a transmission electron microscope (JEM-100 CX II). Thermal 


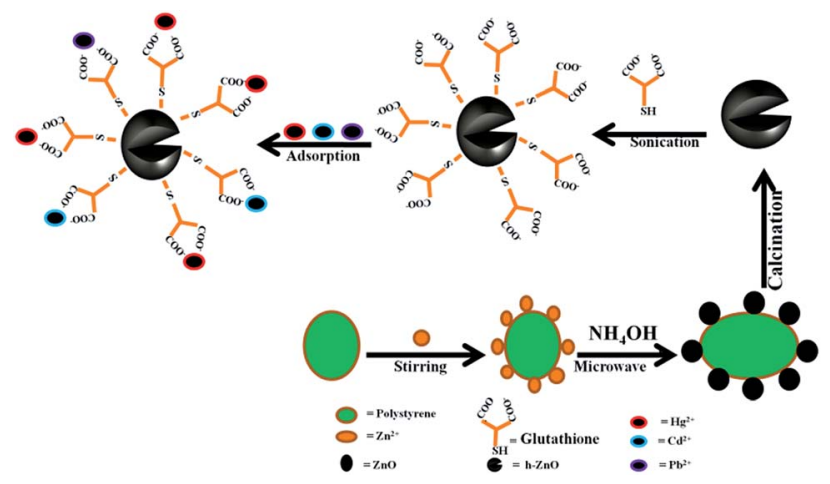

Scheme 1 Schematic representation of the synthesis of Gluah-ZnO and its use as an adsorbent for heavy metal ions.

analysis (TGA/DTA) was carried out using a Simultaneous Thermal Analyzer (STA, Linseis, USA 6807/8835/16). An accurately weighed sample was placed in an alumina crucible and heated from room temperature to $973 \mathrm{~K}$ at a heating rate of 283 $\mathrm{K} \mathrm{min}^{-1}$. The size distributions and zeta potential ( $\left.\zeta\right)$ of the particles were determined with an Anton Paar Litesizer 500 by the DLS technique. Before DLS was carried out, each sample was sonicated for 10 minutes to obtain a fine suspension of the samples for better results. The surface area of the samples was measured by nitrogen adsorption-desorption isotherms using the Brunauer-Emmett-Teller (BET; Micromeritics ASAP 2020) method.

\subsection{Adsorption studies}

The batch adsorption experiments were carried out with $0.020 \mathrm{~g}$ of composite material and $20 \mathrm{ml}$ of $30 \mathrm{ppm}$ aqueous solutions of $\mathrm{Hg}^{2+}, \mathrm{Cd}^{2+}$, and $\mathrm{Pb}^{2+}$ at room temperature and neutral $\mathrm{pH}$. The suspensions were shaken for $6 \mathrm{~h}$ to achieve adsorption equilibration. The suspensions were filtered and the concentrations of the metal ions in the filtrate were determined using an atomic absorption spectrometer (PerkinElmer, AAnalyst 800). The adsorption percentage of metal ions on the composite material was calculated using eqn (1).

$$
\text { Adsorption percentage }=\left(\frac{C_{0}-C_{\mathrm{e}}}{C_{0}}\right) \times 100
$$

The affinity of Glu@h-ZnO towards the metal ions can also be expressed in terms of the distribution coefficient $\left(K_{\mathrm{d}}\right)$, calculated by using eqn (2), and the equilibrium adsorption capacity $\left(q_{\mathrm{e}}\right)$ of the material for a particular ion is obtained using eqn (3).

$$
\begin{gathered}
K_{\mathrm{d}}=\left(C_{0}-C_{\mathrm{e}} / C_{\mathrm{e}}\right) \frac{V}{m}\left(\mathrm{~mL} \mathrm{~g}^{-1}\right) \\
q_{\mathrm{e}}=10^{-3} \times\left(C_{0}-C_{\mathrm{e}}\right) \times \frac{V}{m}\left(\mathrm{mg} \mathrm{g}^{-1}\right)
\end{gathered}
$$

where $V$ is the volume of the metal ion solution (in $\mathrm{ml}$ ), $C_{0}$ is the initial concentration of metal ions, $C_{\mathrm{e}}$ is the concentration of metal ions after adsorption when equilibrium is attained and $m$ is the mass of the adsorbent (in g).

\subsection{Kinetic studies}

Kinetic studies were carried out for $\mathrm{Hg}^{2+}$ at $298 \mathrm{~K}$ with various adsorption contact times (10-280 $\mathrm{min})$. For each operation, $0.020 \mathrm{~g}$ of solid sample was taken into a $50 \mathrm{ml}$ flask containing $20 \mathrm{ml}$ of $30 \mathrm{ppm}$ aqueous solution of $\mathrm{Hg}^{2+}$. At specified time intervals, the suspensions were centrifuged and the ion concentration left in the solution phase after adsorption was determined by AAS.

\subsection{Computational studies}

To study the interaction between the composite material and metal ions, viz., $\mathrm{Cd}^{2+}, \mathrm{Pb}^{2+}$, and $\mathrm{Hg}^{2+}$, the Glu@h-ZnO-metal ion complexes were optimized by density functional theory (DFT) using LanL2DZ and B3LYP as the basis set and functional, respectively. A single point energy (SPE) scan was carried out in order to give a comparative account of the interactions between the composite and the corresponding metal ions, which was expressed in terms of interaction energy, as calculated using eqn (4):

$\Delta E=\left[E_{\mathrm{Glu} @ \mathrm{~h}-\mathrm{ZnO}}+E_{\text {metal ion }}\right]-E_{\mathrm{Glu} @ \mathrm{~h}-\mathrm{ZnO}-\text { metal ion }}\left(\mathrm{kJ} \mathrm{mol}^{-1}\right)(4)$

where $E_{\mathrm{Glu} @ \text { h-ZnOO}}, E_{\text {metal }}$ ion and $E_{\mathrm{Glu} @ \text { h-ZnO-metal ion }}$ are the energy of the composite material, metal ion and composite material-metal ion complex, respectively.

\section{Results and discussion}

\subsection{FTIR analysis}

FTIR spectra of reduced glutathione, h-ZnO and Glu@h-ZnO were recorded in order to confirm the purity of these materials. There are two types of $\mathrm{OH}$ bands present in the FTIR spectrum for $\mathrm{ZnO}$, one corresponding to $-\mathrm{OH}$ groups attached to the metal ions and the other attached to the surfaces..$^{32}$ In the FTIR spectrum of h-ZnO (Fig. 1), the peaks at around $511 \mathrm{~cm}^{-1}$, $1130 \mathrm{~cm}^{-1}$, and $3440 \mathrm{~cm}^{-1}$ are attributed to $\mathrm{Zn}-\mathrm{O}$ deformation, $\mathrm{ZnO}$ stretching, and $\mathrm{O}-\mathrm{H}$ stretching, respectively. The two other prominent peaks at around $1460 \mathrm{~cm}^{-1}$ and $1630 \mathrm{~cm}^{-1}$ in the case of $\mathrm{ZnO}$ and the composite material are due to $\mathrm{O}-\mathrm{H}$ groups attached to metal ions and to the surfaces. ${ }^{39,40}$
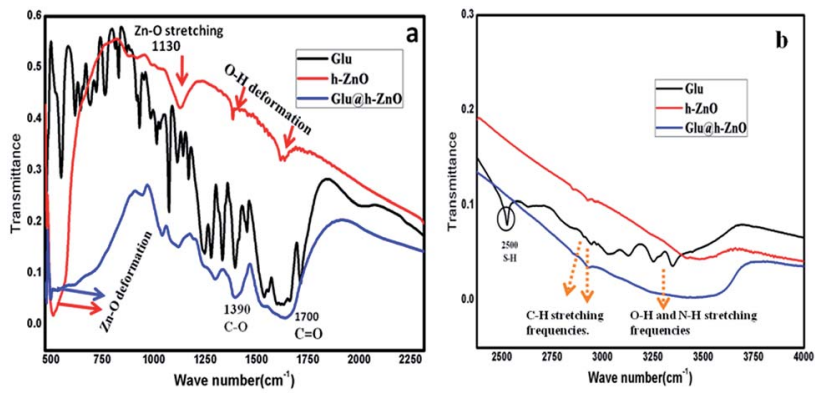

Fig. 1 FTIR spectra of glutathione-coated hollow zinc oxide, reduced glutathione and hollow zinc oxide in the frequency range of $450 \mathrm{~cm}^{-1}$ to $2350 \mathrm{~cm}^{-1}$ (a) and from $2350 \mathrm{~cm}^{-1}$ to $4000 \mathrm{~cm}^{-1}$ (b). 
The IR band at around $1700 \mathrm{~cm}^{-1}$ corresponding to the $>\mathrm{C}=\mathrm{O}$ stretching frequency in addition to the other characteristic bands of the $-\mathrm{COOH}$ group at around $1300 \mathrm{~cm}^{-1}$ and $1400 \mathrm{~cm}^{-1}$ are present in the FTIR spectra of both glutathione and Glu@h-ZnO. Besides, in both of these materials, IR bands at around $2800 \mathrm{~cm}^{-1}$ and $2900 \mathrm{~cm}^{-1}$ are present, which are attributed to the methyl stretching frequencies. The presence of such characteristic peaks of glutathione in the composite material suggests a possible coating of glutathione over the hollow ZnO surface. Furthermore, a prominent IR band at around $2500 \mathrm{~cm}^{-1}$, corresponding to $\mathrm{S}-\mathrm{H}$ deformation, is present in the reduced glutathione but not in the Glu@h-ZnO spectrum. The diminishing of the S-H peak may be because glutathione binds to h-ZnO through sulfur, as a result of which the existence of an $\mathrm{S}-\mathrm{H}$ bond in the composite is highly unlikely.

\subsection{Thermal gravimetric (TGA) analysis}

Thermal analysis, in terms of TGA and DTA, was carried out for the composite material as well as for the individual components for comparison purposes (Fig. 2). In the case of pure $\mathrm{ZnO}$, weight loss occurs up to $200{ }^{\circ} \mathrm{C}$ and thereafter it remains constant. This weight loss is attributed to the removal of surface-bound water and the decomposition of $\mathrm{Zn}(\mathrm{OH})_{2} \cdot{ }^{41,42}$ Contrary to this, an extra weight loss stage is observed in case of Glu@h-ZnO in the temperature range of 270-580 ${ }^{\circ} \mathrm{C}$, which is attributed to the decomposition of glutathione. It is important to note that pure glutathione starts to decompose at around $200{ }^{\circ} \mathrm{C}$, as can be seen from its thermogram. Delayed decomposition of glutathione in the case of Glu@h-ZnO indicates that its thermal stability is enhanced after it is coated over the ZnO surface. The same thing is also reflected by the DTA results. Endothermic peaks at around $100{ }^{\circ} \mathrm{C}$ in the case of $\mathrm{ZnO}$ and Glu@h-ZnO indicate the loss of water. Another endothermic peak at $270{ }^{\circ} \mathrm{C}$ in the case of Glu@h-ZnO represents the melting of glutathione. However, the same peak in the case of pure glutathione appears at $200{ }^{\circ} \mathrm{C}$, which indicates a substantial improvement in the thermal stability of Glu@h-ZnO. Exothermic peaks in the temperature range of $380-450{ }^{\circ} \mathrm{C}$ for pure glutathione and Glu@h-ZnO represent oxidation of glutathione. Exothermic peaks in the temperature range of $470-$
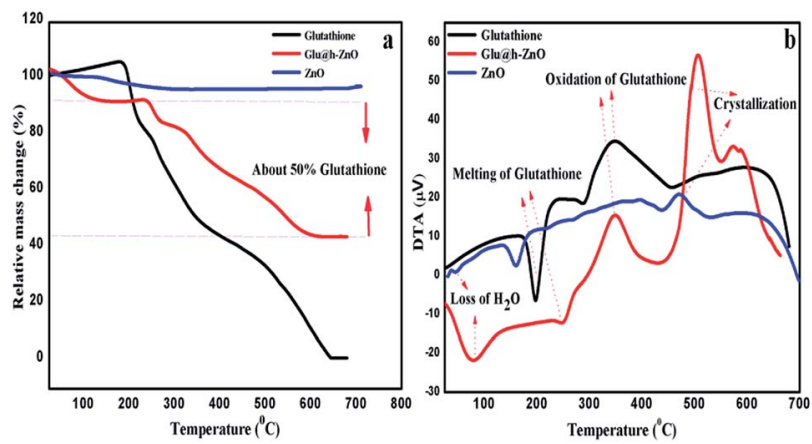

Fig. 2 TGA (a) and DTA (b) curves of $\mathrm{h}-\mathrm{ZnO}$, glutathione and Gluah$\mathrm{ZnO}$.
$530{ }^{\circ} \mathrm{C}$ for $\mathrm{ZnO}$ and Glu@h-ZnO may be attributed to crystallization of the amorphous part of $\mathrm{ZnO}^{43}$

Further, from the TGA curve of Glu@h-ZnO, it can be inferred that there is approximately 50\% weight loss due to glutathione. The value is close to the $1: 1$ ratio of h-ZnO and glutathione taken at the time of its synthesis.

\subsection{Microscopic studies}

The SEM images of h-ZnO (Fig. 3a-d) clearly show that ZnO particles are hollow and some big cavities are visible in the SEM images at higher magnification. Furthermore, the surface of the uncoated hollow particles appears to be smooth with very little agglomeration of particles. On the other hand, the Glu@h-ZnO composite material appears as agglomerated and the hollowness of the particles is not quite visible in the SEM images (Fig. 3e and f). Agglomeration and diminishing of the hollow character in the case of Glu@h-ZnO may be due to the extensive coating of glutathione over the hollow $\mathrm{ZnO}$ particles.

Fig. 4 shows the TEM images of hollow ZnO and Glu@h-ZnO at different magnifications. The surface of the uncoated hollow ZnO (Fig. 4a and b) appears to be smoother in the TEM images compared to the surface of the coated particles (Fig. 4c-f). From TEM analysis, the average size of the Glu@h-ZnO particles is around $35 \mathrm{~nm}$. A glutathione coating of approximately $11 \mathrm{~nm}$ thickness can be seen over the surface of a h-ZnO particle (inset Fig. 4f). The glutathione coating has a drastic effect in screening the hollow character of the $\mathrm{ZnO}$ particles as the cavities are occupied by glutathione molecules as well. As a result, the hollowness of the particles is not quite visible in the TEM images. The diameter of the cavity is not uniform and varies from particle to particle. The biggest cavity is about $34 \mathrm{~nm}$ in diameter.

\subsection{XRD analysis}

Fig. 5 shows the XRD pattern for the uncoated $\mathrm{h}-\mathrm{ZnO}$ and Glu@h-ZnO. It can be seen that the peak intensity in the XRD pattern of $\mathrm{h}-\mathrm{ZnO}$ is sharp and narrow, confirming that the sample is of high quality with good crystallinity. The lines at $2 \theta$ values equal to $31.76^{\circ}, 34.58^{\circ}$, and $36.67^{\circ}$ are due to reflection from the crystallographic (100), (002), and (101) planes, respectively, while the peaks at $47.9^{\circ}, 57.02^{\circ}, 63.3^{\circ}, 66.68^{\circ}$,

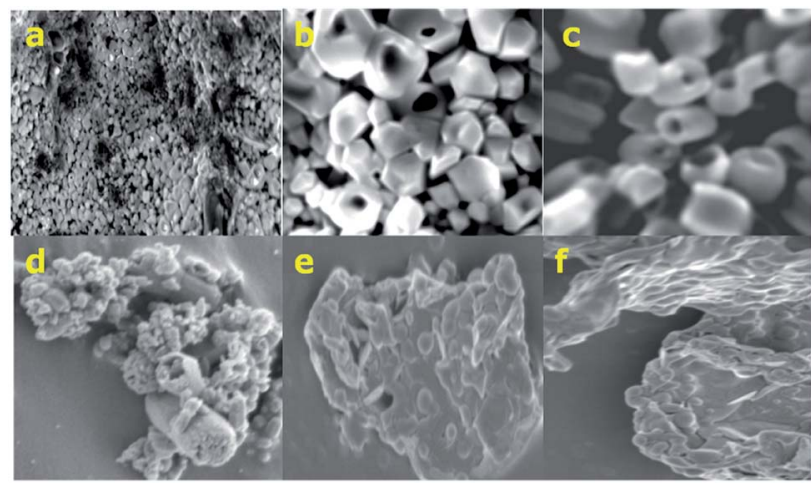

Fig. 3 SEM images of $\mathrm{h}-\mathrm{ZnO}(\mathrm{a}-\mathrm{d})$ and Glu@h-ZnO (e and f). 


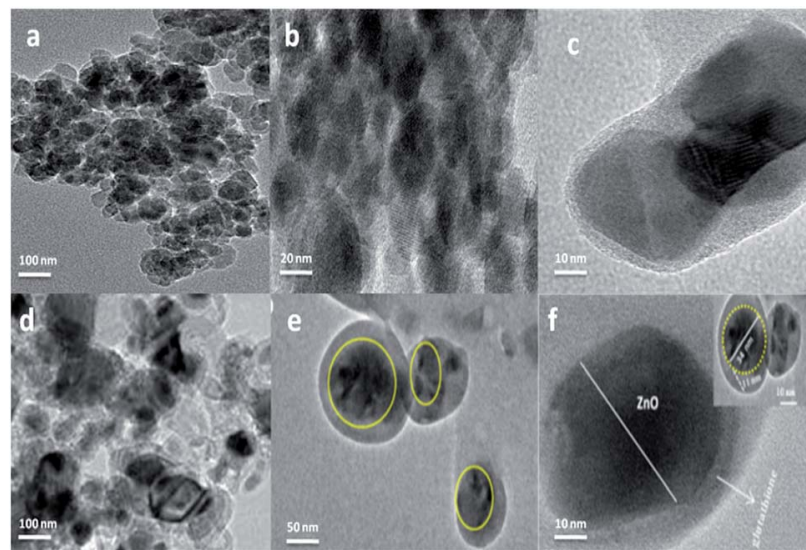

Fig. 4 TEM images of hollow $\mathrm{ZnO}(\mathrm{a}$ and $\mathrm{b})$ and Gluah- $\mathrm{ZnO}(\mathrm{c}-\mathrm{f})$.

$68.26^{\circ}$ and $69.36^{\circ}$ correspond to the $102,110,103,200,112$ and 201 planes, respectively, and they are in accordance with the wurtzite structure of $\mathrm{ZnO}$ (International Centre for Diffraction Data, JCPDS 5-0664). No impurity peaks were observed, suggesting that high-purity $\mathrm{ZnO}$ was obtained. The XRD pattern of Glu@h-Zno has similar peaks except the peak intensity is reduced and the low intensity peak corresponding to the (200) plane is absent in the composite when compared with pure h$\mathrm{ZnO}$. The reduction in the peak intensity ${ }^{\mathbf{4}}$ and the absence of the peak pertaining to the 200 plane indicate the successful coating of glutathione over the h-ZnO particles. The peak intensity is sharp, even in the composite, which may be due to the high crystallinity acquired as the outcome of the calcination during the formation of the h-ZnO.

\subsection{DLS measurements}

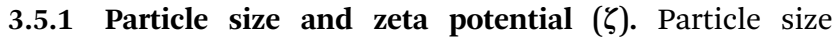
distribution by intensity was obtained for both h-ZnO and Glu@h-ZnO. The particle size measurements for Glu@h-ZnO at pH 7 show that it has a hydrodynamic diameter of $1483.50 \mathrm{~nm}$ and PDI of $17.3 \%$. The PDI value of greater than $10 \%$ indicates the heterogeneous nature of particles, which may be attributed to the non-uniform coating of glutathione over the $\mathrm{h}-\mathrm{ZnO}$ particles owing to different sized cavities in the h-ZnO. The effect of $\mathrm{pH}$ on the hydrodynamic diameter of the Glu@h-ZnO particles was also determined (Fig. S1a $\dagger$ ). It was found that

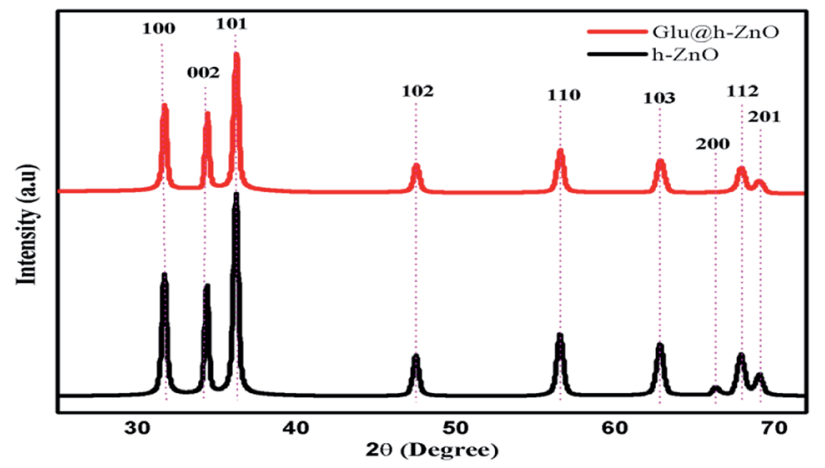

Fig. 5 XRD patterns of Gluah- $\mathrm{ZnO}$ and $\mathrm{h}-\mathrm{ZnO}$. the hydrodynamic diameter showed a continuous decrease when the $\mathrm{pH}$ was increased from 4 to 8 . This is because, even at $\mathrm{pH} 4$, the surface of Glu@h-ZnO is sufficiently negative and when the $\mathrm{pH}$ is increased, more and more $-\mathrm{COOH}$ groups of glutathione are de-protonated, making the surface more negatively charged (as revealed from the zeta potential studies). The higher the magnitude of the charge on the surface, the more strongly they will repel, thus preventing the possibility of agglomeration. ${ }^{45}$ On the other hand, a hydrodynamic diameter of $8197 \mathrm{~nm}$ at neutral $\mathrm{pH}$ was obtained for h-ZnO from DLS studies (Fig. S1b $\dagger$ ) with a polydispersity index (PDI) of $9.7 \%$ (less than $10 \%$ ) indicating the monodispersed nature of the material.

Zeta potential measurements were carried out for both Glu@h-ZnO and uncoated h-ZnO (Fig. S2 $\dagger$ ). The $\zeta$ of uncoated $\mathrm{h}-\mathrm{ZnO}$ was found to be only $-3.1 \mathrm{mV}$ at neutral $\mathrm{pH}$, which is very small compared to the $\zeta(-21.72 \mathrm{mV})$ of Glu@h-ZnO under the same conditions. The effect of $\mathrm{pH}$ on the $\zeta$ was also determined for Glu@h-ZnO. It can be seen that at $\mathrm{pH} 4$, the $\zeta$ of Glu@h-ZnO is -6.72 , which may be due to some surface-bound -OH groups and glutathione molecules remaining deprotonated even at $\mathrm{pH}$. When the $\mathrm{pH}$ is increased from 4 to 8 , more and more $-\mathrm{COOH}$ groups of glutathione and surfacebound $-\mathrm{OH}$ groups are de-protonated, making the surface more negative. The variation of the $\zeta$ and hydrodynamic diameter of Glu@h-ZnO with pH is summarized in Table 1 and it can be seen that there is an inverse relationship between surface charge and hydrodynamic diameter.

\subsection{BET studies}

The surface area of the material plays a large role in the process of adsorption of metal ions. The higher the surface area, the more sites will be available for the metal ions for adsorption to take place. In this regard, we evaluated the surface area of both hollow ZnO and Glu@h-ZnO using the nitrogen adsorptiondesorption method at $77 \mathrm{~K}$. The $\mathrm{N}_{2}$ adsorption-desorption isotherms in the relative pressure range of 0 to 1 are shown in Fig. 6. The surface areas calculated for hollow ZnO and Glu@h$\mathrm{ZnO}$ using BET isotherms were found to be $17.1 \mathrm{~m}^{2} \mathrm{~g}^{-1}$ and 12.3 $\mathrm{m}^{2} \mathrm{~g}^{-1}$, respectively. The BET surface area calculated for hollow $\mathrm{ZnO}$ is much higher when it is compared to the normal $\mathrm{ZnO}{ }^{46}$ This indicates that glutathione coating over hollow $\mathrm{ZnO}$ will have a prominent effect in promoting the adsorption capability of Glu@h-ZnO towards heavy metal ions. Furthermore, when the surface area of uncoated and coated hollow $\mathrm{ZnO}$ was compared, a significant decrease was observed in the case of the latter. This decrease in surface area may be attributed to the occupation of cavities by glutathione molecules during the process of coating.

\subsection{Adsorption studies}

3.7.1 Heavy metal removal. The glutathione molecules present on the surface of the h-ZnO have a good tendency to bind with the metal ions, so the metal ion removal characteristics of Glu@h-ZnO were investigated by carrying out adsorption experiments with individual solutions of $\mathrm{Pb}^{2+}, \mathrm{Cd}^{2+}$, and $\mathrm{Hg}^{2+}$, as shown in Table 2 . 
Table 1 Variation of $\zeta$ and the hydrodynamic diameter of Gluah-ZnO particles with $\mathrm{pH}$

\begin{tabular}{lcccc}
\hline $\mathrm{pH}$ & 4 & 5 & 6 & 7 \\
\hline Zeta potential (mV) & -6.72 & -10.55 & -13.62 & -21.72 \\
Hydrodynamic diameter (nm) & 2626 & 1823 & 1483 & -23.39 \\
\hline
\end{tabular}

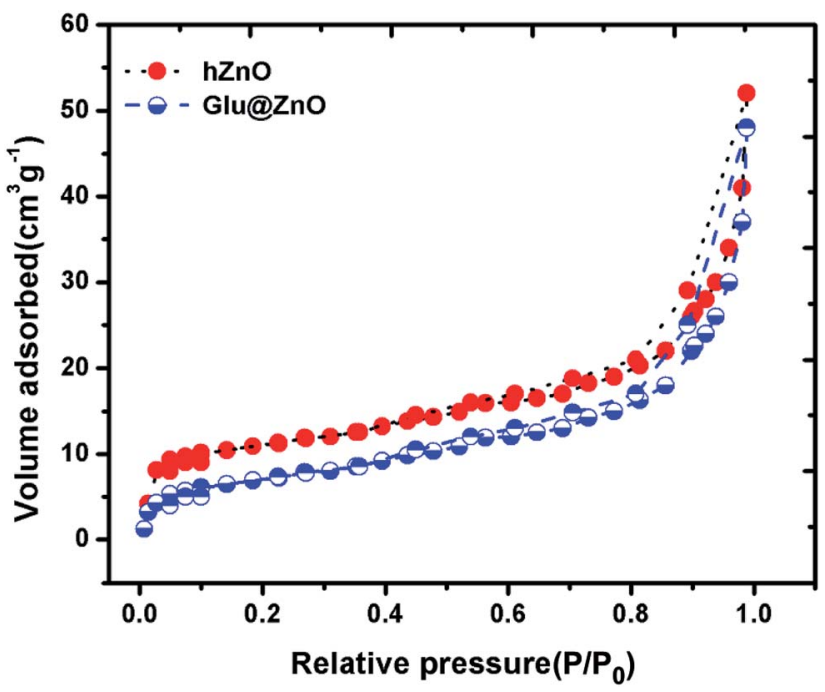

Fig. 6 Nitrogen adsorption-desorption isotherms of hollow $\mathrm{ZnO}$ and the Gluah-ZnO composite recorded at $77 \mathrm{~K}$.

It can be seen from the table that the concentrations of these ions decrease from the starting value of $\sim 30 \mathrm{ppm}$ and the maximum decrease was obtained in the case of $\mathrm{Hg}^{2+}$, indicating that the adsorption ability of Glu@h-ZnO towards $\mathrm{Hg}^{2+}$ is higher than those for $\mathrm{Cd}^{2+}$ and $\mathrm{Pb}^{2+}$ ions.

The affinity of Glu@h-ZnO towards the uptake of these metal ions follows the order: $\mathrm{Hg}^{2+}>\mathrm{Cd}^{2+}>\mathrm{Pb}^{2+}$, as shown in Fig. $\mathrm{S} 3, \dagger$ which is the same as previously reported by our group ${ }^{47}$ and is also in good agreement with the data obtained from the present DFT studies. As the material shows the highest affinity towards the uptake of $\mathrm{Hg}^{2+}$ ions, this ion was selected for further studies.

3.7.2 Effect of pH on adsorption. Metal ions in aqueous solution can be present in various forms at different $\mathrm{pH}$ values. Therefore, the $\mathrm{pH}$ of the solution plays an important role in the adsorption of metal ions on Glu@h-ZnO. The adsorption of metal ions on Glu@h-ZnO was investigated at $\mathrm{pH}$ values from 4

Table 2 Percentage removal and $K_{d}$ values of $\mathrm{Pb}^{2+}, \mathrm{Cd}^{2+}$, and $\mathrm{Hg}^{2+}$ ions by Gluah- $\mathrm{ZnO}$ at $\mathrm{pH}=7, T=298 \mathrm{~K}, m=0.02 \mathrm{~g}, \mathrm{~V}=20 \mathrm{ml}$, conc. of metal ion $=\sim 30 \mathrm{ppm}$ and contact time $=6 \mathrm{~h}$

\begin{tabular}{lllll}
$\begin{array}{l}\text { Metal } \\
\text { ion }\end{array}$ & $C_{0}(\mathrm{ppm})$ & $C_{\mathrm{e}}(\mathrm{ppm})$ & Metal ion removal $(\%)$ & $K_{\mathrm{d}}\left(\mathrm{ml} \mathrm{g}^{-1}\right)$ \\
\hline $\mathrm{Pb}^{2+}$ & 29.75 & 4.97 & 83.29 & $9.8 \times 10^{3}$ \\
$\mathrm{Cd}^{2+}$ & 30.15 & 4.05 & 86.57 & $1.3 \times 10^{4}$ \\
$\mathrm{Hg}^{2+}$ & 29.36 & 1.12 & 96.18 & $5.0 \times 10^{4}$
\end{tabular}

to 8. As shown in Fig. 7, the adsorption of $\mathrm{Hg}^{2+}$ increases continuously as the $\mathrm{pH}$ increases from 4 to 8 .

At pH 4 only about $40 \%$ of $\mathrm{Hg}^{2+}$ is removed, but the removal reaches about $97 \%$ at a $\mathrm{pH}$ of 8 . The mode of interaction is expected to be electrostatic ${ }^{48}$ between the metal ions and $-\mathrm{COO}^{-}$groups of Glu@h-ZnO and the same is revealed from the present DFT studies and pH experiments. The surface charge of Glu@h-ZnO is negative in the $\mathrm{pH}$ range of 4 to 8 with a higher magnitude at higher $\mathrm{pH}$ values, as revealed from DLS studies. This means that a $\mathrm{pH}$ higher than 4 is beneficial for the ionization of the oxygen-containing functional groups on the surface of Glu@h-ZnO, which play a significant role in the uptake of metal ions. The negative charges generated on the Glu@h-ZnO surface enhance the adsorption capacity of Glu@h$\mathrm{ZnO}$ and, simultaneously, the electrostatic attraction becomes more important. The adsorption of metal ions decreases at lower $\mathrm{pH}$ values owing to the low dissociation of the functional groups and competition between $\mathrm{H}^{+}$ions and metal ions for the same sorption sites. Although there is greater adsorption of metal ions at higher $\mathrm{pH}$ values, we carried out further studies at neutral $\mathrm{pH}$ because at basic $\mathrm{pH}$ most of the metal ions are precipitated and also because the $\mathrm{pH}$ of the aqueous systems happens to be around 7 .

3.7.3 Batch adsorption studies for $\mathrm{Hg}^{2+}$ ion uptake. From the results described above, Glu@h-ZnO shows the highest selectivity for the removal of $\mathrm{Hg}^{2+}$. The maximum adsorption capacity of the material was determined from an adsorption equilibrium study. The $\mathrm{Hg}^{2+}$ uptake by Glu@h-ZnO was found to increase successively with increasing concentration (10-600 ppm) of $\mathrm{Hg}^{2+}$ ions, as shown in Table 3 .

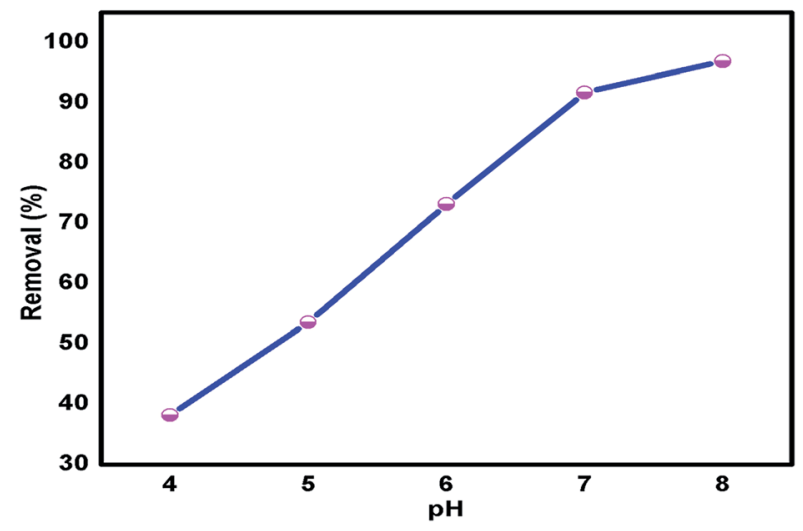

Fig. 7 Variation of $\mathrm{Hg}^{2+}$ ion adsorption by Gluah- $\mathrm{ZnO}$ at various $\mathrm{pH}$ values at $T=298 \mathrm{~K}$, mass of adsorbent $(\mathrm{m})=0.020 \mathrm{~g}$ and volume of metal ion solution $(V)=20 \mathrm{ml}$. 
The percent ion removal, $q_{\mathrm{e}}$ and $K_{\mathrm{d}}$ values over this wide range of initial concentration (10-600 ppm) of metal ion were obtained using eqn (1), (2) and (3), respectively. The maximum adsorption capacity $\left(q_{\mathrm{m}}\right)$ of Glu@h-ZnO for $\mathrm{Hg}^{2+}$ was found to be around $233 \mathrm{mg} \mathrm{g}^{-1}$ (Fig. 8a), beyond which no appreciable change in the $q_{\mathrm{e}}$ value was found.

This $q_{\mathrm{m}}$ value for Glu@h-ZnO is quite satisfactory when compared to the $q_{\mathrm{m}}$ values of other materials reported in the literature (Table 4). To correlate the amount of $\mathrm{Hg}^{2+}$ ions adsorbed by unit mass of adsorbent, the Langmuir adsorption isotherm ${ }^{49}$ was used. In this model, the adsorbate moieties $\left(\mathrm{Hg}^{2+}\right.$ ions $)$ are assumed to undergo monolayer type coverage over energetically identical adsorption sites of the adsorbent surface. This model assumes that no further adsorption can take place at a site that is already occupied by the adsorbate moiety. The Langmuir isotherm model can be written as shown in eqn (5):

$$
\frac{1}{q_{\mathrm{e}}}=\frac{1}{q_{\mathrm{m}}}+\frac{1}{b q_{\mathrm{m}} C_{\mathrm{e}}}
$$

where $q\left(\mathrm{mg} \mathrm{g}^{-1}\right)$ represents the equilibrium adsorption capacity of $\mathrm{Hg}^{2+}$ ions adsorbed, $C_{\mathrm{e}}\left(\mathrm{mg} \mathrm{L}^{-1}\right)$ is the $\mathrm{Hg}^{2+}$ ion concentration at equilibrium, $q_{\mathrm{m}}\left(\mathrm{mg} \mathrm{g}^{-1}\right)$ is the theoretical maximum adsorption capacity and $b$ is the Langmuir constant. The values of $q_{\mathrm{m}}$ and $b$ were obtained from the intercept and the slope of the linear plot of $1 / q_{\mathrm{e}} v s .1 / C_{\mathrm{e}}$ (Fig. 8b), respectively. The theoretical maximum adsorption capacity $(q \mathrm{~m}, \mathrm{cal})$ was found to be $222.22 \mathrm{mg} \mathrm{g}^{-1}$, which is very close to the experimental maximum adsorption capacity $\left(q_{\mathrm{m}, \exp }=233 \mathrm{mg} \mathrm{g}^{-1}\right)$ given in Table 5 .

Furthermore, the correlation coefficient being close to $1\left(R^{2}\right.$ $=0.9888$ ) suggests the adsorption of $\mathrm{Hg}^{2+}$ ions on the surface of Glu@h-Zno is monolayer and can be well described by the Langmuir model. The separation factor $\left(R_{\mathrm{L}}\right)$ was evaluated by using eqn (6) to determine whether the adsorption process is linear $\left(R_{\mathrm{L}}=1\right)$, unfavourable $\left(R_{\mathrm{L}}>1\right)$, favourable $\left(0<R_{\mathrm{L}}<1\right)$ or irreversible $\left(R_{\mathrm{L}}=0\right) .{ }^{58}$ In our case, the value of $R_{\mathrm{L}}$ was 0.225 , indicating the favourable adsorption of $\mathrm{Hg}^{2+}$ on Glu@h-ZnO.

$$
R_{\mathrm{L}}=\frac{1}{1+b C_{0}}
$$

Table 3 Removal percentage, adsorption capacity (q) and distribution coefficient $\left(K_{\mathrm{d}}\right)$ value of Gluah-ZnO towards the uptake of different $\mathrm{Hg}^{2+}$ ion concentrations at $\mathrm{pH}=7, T=298 \mathrm{~K}, m=0.02 \mathrm{~g}, \mathrm{~V}=20 \mathrm{ml}$,

\begin{tabular}{|c|c|c|c|c|}
\hline$C_{0}(\mathrm{ppm})$ (approx.) & $C_{\mathrm{e}}(\mathrm{ppm})$ & Removal (\%) & $q_{\mathrm{e}}\left(\mathrm{mg} \mathrm{g}^{-1}\right)$ & $K_{\mathrm{d}}\left(\mathrm{ml} \mathrm{g}^{-1}\right)$ \\
\hline 10 & 0.31 & 96.9 & 9.69 & $3.1 \times 10^{4}$ \\
\hline 40 & 1.92 & 95.2 & 38.08 & $2.0 \times 10^{4}$ \\
\hline 80 & 7.74 & 90.32 & 72.26 & $9.3 \times 10^{3}$ \\
\hline 150 & 20.97 & 86.02 & 129.03 & $6.2 \times 10^{3}$ \\
\hline 220 & 31.44 & 85.71 & 188.56 & $6.0 \times 10^{3}$ \\
\hline 300 & 80.35 & 73.21 & 219.65 & $2.7 \times 10^{3}$ \\
\hline 400 & 167.80 & 58.05 & 232.2 & $1.4 \times 10^{3}$ \\
\hline 600 & 366.98 & 38.83 & 233.02 & $6.3 \times 10^{2}$ \\
\hline
\end{tabular}
$\mathrm{V} / \mathrm{m}=1000 \mathrm{ml} \mathrm{g}^{-1}$ and contact time $=6 \mathrm{~h}$
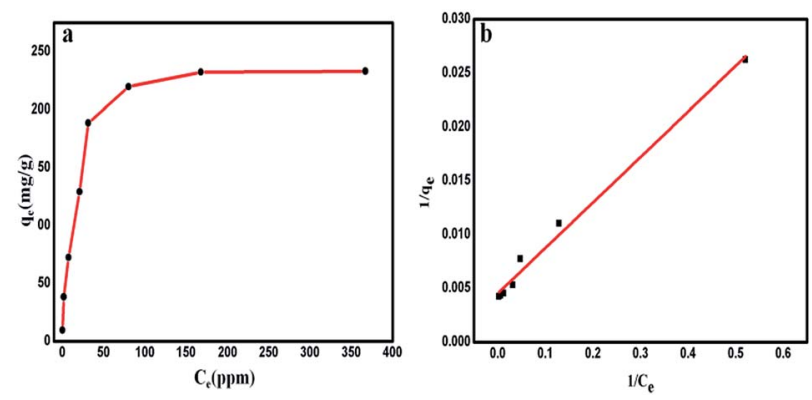

Fig. 8 Variation of the equilibrium adsorption capacity $\left(q_{\mathrm{e}}\right)$ of Gluah$\mathrm{ZnO}$ towards the uptake of $\mathrm{Hg}^{2+}$ solutions of different concentrations (10-600 ppm) at $\mathrm{pH}=7$ and $T=298 \mathrm{~K}$ (a). The Langmuir adsorption isotherm model for the adsorption of $\mathrm{Hg}^{2+}$ ions on Gluah- $\mathrm{ZnO}$ (b).

where $C_{0}$ is the lowest initial concentration of metal ions and $b$ is the Langmuir adsorption constant.

3.7.4 Adsorption kinetics. It is very significant to investigate the adsorption kinetics in order to study the adsorption rate and pathways of adsorption until equilibrium is reached. Adsorption kinetic experiments for $\mathrm{Hg}^{2+}$ at various adsorption contact times (5-280 min) were performed and it can be seen from Fig. 9A that the rate of adsorption is very fast in the first 40-50 minutes but thereafter it slows down and equilibrium is attained after a contact time of 100 minutes. The rate of adsorption was determined by using two different rate equations, pseudo-first order and pseudo-second order, and a comparison was drawn between the experimental and calculated data, as shown in Table 6. The two kinetic rate equations can be written as follows..$^{59}$

Pseudo-first order

$$
\ln \left(q_{\mathrm{e}}-q_{t}\right)=\ln q_{\mathrm{e}}-k_{1} t
$$

\begin{tabular}{|c|c|c|c|}
\hline S. no & Adsorbent & $q_{\mathrm{m}}\left(\mathrm{mg} \mathrm{g}^{-1}\right)$ & Ref. \\
\hline 1 & $\mathrm{GAC}^{a}$ & 20.83 & 50 \\
\hline 2 & MNPs-DTC ${ }^{b}$ & 47.87 & 51 \\
\hline 3 & $\mathrm{CSTG}^{c}$ & 98 & 52 \\
\hline 4 & PANI-PS $^{d}$ composite & 148 & 53 \\
\hline 5 & $\begin{array}{l}\text { Mercaptosuccinic acid- } \\
\mathrm{LDH}^{e}\end{array}$ & 161 & 54 \\
\hline 6 & $\mathrm{PAM} / \mathrm{ATP}^{f}$ & 192 & 55 \\
\hline 7 & Glu@h-Zno $g$ & 233 & Current work \\
\hline 8 & KMS- $2^{h}$ & 297 & 56 \\
\hline 9 & KMS-1 ${ }^{i}$ & 377 & 57 \\
\hline 10 & $\mathrm{MoS}_{4}-\mathrm{LDH}^{j}$ & 500 & 18 \\
\hline \multicolumn{4}{|c|}{$\begin{array}{l}{ }^{a} \text { Carboxymethylated granular activated carbon (GAC). }{ }^{b} \text { Magnetic } \\
\text { nanoparticles (MNPs) of } \mathrm{Fe}_{3} \mathrm{O}_{4} \text { functionalized by dithiocarbamate } \\
\text { (DTC). }{ }^{c} \text { Chitosan-thioglyceraldehyde Schiff's base cross-linked } \\
\text { magnetic resin (CSTG). }{ }^{d} \text { Polyaniline-polystyrene composite (PANI- } \\
\text { PS). }{ }^{e} \text { Mercaptocarboxylic acid intercalated Mg-Al layered double } \\
\text { hydroxide. Polyacrylamide/attapulgite (PAM/ATP). }{ }^{g} \text { Glutathione- } \\
\text { coated hollow ZnO (Glu@h-ZnO). }{ }^{h} \text { Layered } \mathrm{K}_{2} \mathrm{MgSn} \mathrm{C}_{2} \mathrm{~S}_{6} \text { (KMS-2). } \\
{ }^{i} \text { Layered metal sulfides (KMS-1). }{ }^{j} \text { Layered double hydroxide } \\
\text { intercalated with the } \mathrm{MoS}_{4}{ }^{2-} \text { ion. }\end{array}$} \\
\hline
\end{tabular}

Table 4 A comparison of the adsorption capacities of various adsorbents for $\mathrm{Hg}^{2+}$ ions 
Table 5 Adsorption isotherm constants for the adsorption of $\mathrm{Hg}^{2+}$ by Gluah-ZnO at $T=298 \mathrm{~K}$

\begin{tabular}{llll}
\hline$q_{\mathrm{m}, \mathrm{cal}}\left(\mathrm{mg} \mathrm{g}^{-1}\right)$ & $b\left(\mathrm{~L} \mathrm{mg}^{-1}\right)$ & $R^{2}$ & $R_{\mathrm{L}}$ \\
\hline 222.22 & 0.34 & 0.9888 & 0.225
\end{tabular}

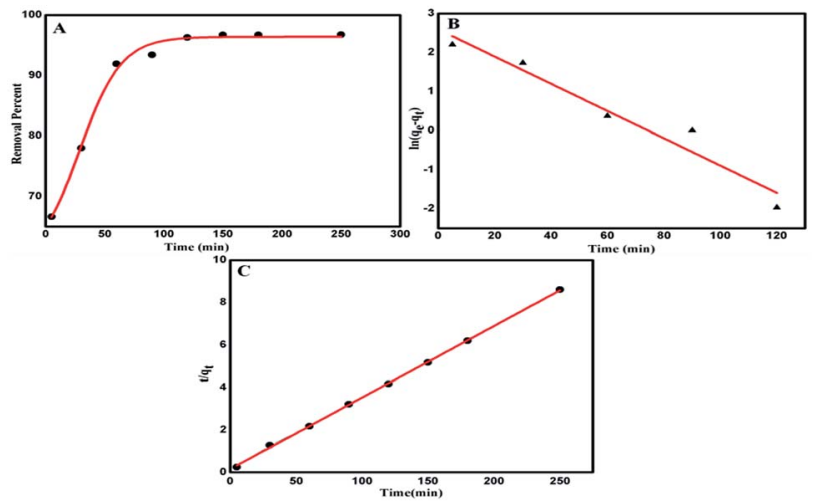

Fig. 9 Removal percentage of $\mathrm{Hg}^{2+}$ as a function of time (A). Pseudofirst order kinetic plot for $\mathrm{Hg}^{2+}$ ion removal (B) and pseudo-secondorder kinetic plot for $\mathrm{Hg}^{2+}$ ion removal (C).

Pseudo-second order

$$
\frac{t}{q_{t}}=\frac{1}{k_{2} q_{\mathrm{e}}{ }^{2}}+\frac{t}{q_{\mathrm{e}}}
$$

where $q_{\mathrm{e}}\left(\mathrm{mg} \mathrm{g}^{-1}\right)$ and $q_{t}\left(\mathrm{mg} \mathrm{g}^{-1}\right)$ are the amounts of $\mathrm{Hg}^{2+}$ adsorbed at equilibrium and at time $t$, respectively, while $k_{1}$ $\left(\mathrm{min}^{-1}\right)$ and $k_{2}\left(\mathrm{~g} \mathrm{mg}^{-1} \mathrm{~min}^{-1}\right)$ are the equilibrium rate constants of the pseudo-first-order and pseudo-second-order rate equations, respectively.

The value of $k_{1}$ was obtained from the slope of the plot of $\ln \left(q_{\mathrm{e}}-q_{t}\right)$ vs. $t$ (Fig. 9B) while $k_{2}$ was obtained from the intercept of the plot of $t / q_{t} v s$. $t$ (Fig. 9C). The various pseudo-first-order and pseudo second order kinetic parameters are summarized in Table 6 . The correlation coefficient $\left(R^{2}\right)$ corresponding to the pseudo first order model is only 0.9333 but the value is much closer to 1 (being equal to 0.9995 ) for the pseudo second order rate model. Furthermore, it can be seen that the $q_{\mathrm{e}, \mathrm{cal}}$ value is much close to the $q_{\text {e,exp }}$ value for the pseudo-second order model while the two differ marginally for the pseudo-first order model. These results indicate that a pseudo-second order kinetic model is suitable for describing the adsorption of $\mathrm{Hg}^{2+}$ ions on Glu@h-ZnO particles where chemical forces are holding the metal ions and the adsorbent together. ${ }^{60}$

\subsection{Computational studies}

To study the interaction between Glu@h-ZnO and metal ions, viz., $\mathrm{Cd}^{2+}, \mathrm{Pb}^{2+}$, and $\mathrm{Hg}^{2+}$, density functional theory studies were carried out using the LanL2DZ basis set at the B3LYP level of theory. From these theoretical calculations, the optimized geometries and interaction energies of the composite-metal ion systems were obtained. The optimized geometries are given in Fig. 10 and from these computational studies it was found that the most favourable metal ion interaction takes place with the oxygen of the glutathione molecule.

SPE scans were carried out for all the complex models in order to give a comparative account of the interaction for all the complex models studied, as shown in Fig. S4. $\dagger$ The interaction between the composite and the corresponding metal ions was expressed in terms of interaction energy (in $\mathrm{kJ} \mathrm{mol}^{-1}$ ) and was found to be -2218.29 for $\mathrm{Pb}^{2+},-2443.34$ for $\mathrm{Cd}^{2+}$ and -2500.46 for $\mathrm{Hg}^{2+}$. This indicates that Glu@h-ZnO has the highest affinity for $\mathrm{Hg}^{2+}$, followed by $\mathrm{Cd}^{2+}$ and then $\mathrm{Pb}^{2+}$ ions. The computational results are in good congruence with the experimental values obtained from the adsorption studies of the composite material.

\subsection{Proposed mechanism of adsorption}

Metal ion binding to Glu@h-ZnO is preferentially based on electrostatic interactions between the negatively charged groups on Glu@h-ZnO and the positively charged metal ions. Investigation of the effect of $\mathrm{pH}$ on adsorption revealed very low adsorption at low $\mathrm{pH}$ values, mainly because of the diminishing negative charge on the Glu@h-ZnO surface at lower $\mathrm{pH}$ values, as a result of which the ionic interactions become weaker and the adsorption of metal ions is slowed down in acidic medium. The DFT studies also suggested that electrostatic interactions

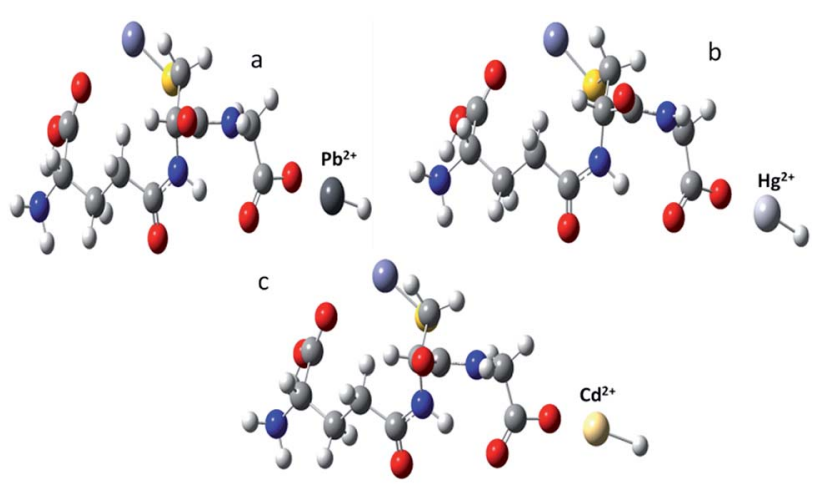

Fig. 10 Optimized geometries of metal ion complexed glutathione obtained from DFT studies: (a) $\mathrm{Pb}^{2+}$; (b) $\mathrm{Hg}^{2+}$; and (c) $\mathrm{Cd}^{2+}$.

Table 6 Kinetic parameters obtained for the adsorption of $\mathrm{Hg}^{2+}$ ions by Gluah- $\mathrm{ZnO}$

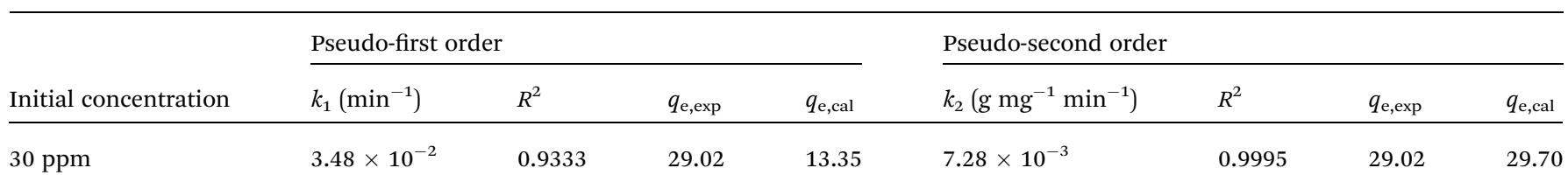


are mainly responsible for the adsorption of metal ions on Glu@h-ZnO. From the DFT studies, it was found that the oxygen atoms of the $-\mathrm{COOH}$ group have more interacting power than the nitrogen atoms present in the glutathione moiety. Computational results for adsorption of metal ions by Glu@h-ZnO indicate the transfer of hydrogen from the $-\mathrm{COOH}$ of glutathione to the metal ion (Fig. 10) for optimized electrostatic interactions between the two. The transfer of $\mathrm{H}^{+}$from the $-\mathrm{COOH}$ group to the metal ion certainly enhances the negative and positive charges on the glutathione and metal ion, respectively, for stronger ionic bonding during adsorption. From this discussion, it can be concluded that pure ionic interactions are responsible for holding the metal ions on Glu@h-ZnO during the process of adsorption.

\section{Conclusions}

Glu@h-ZnO was successfully synthesized under microwave conditions, as confirmed by various characterization techniques. The as-synthesized material was used as an adsorbent for the uptake of $\mathrm{Hg}^{2+}, \mathrm{Cd}^{2+}$, and $\mathrm{Pb}^{2+}$ ions and the maximum uptake was found towards $\mathrm{Hg}^{2+}$ ions, with a maximum adsorption capacity of $233 \mathrm{mg} \mathrm{g}^{-1}$, which is reasonably good for this material. The adsorption of $\mathrm{Hg}^{2+}$ by Glu@h-ZnO follows pseudo-second-order rate kinetics and can be well described by the Langmuir model. Finally, DFT studies were carried out to correlate theoretical results with the experimental data. The computational results so obtained were in good congruence with the experimental values obtained from adsorption studies using the composite material.

\section{Conflicts of interest}

There are no conflicts to declare.

\section{Acknowledgements}

We are thankful to the Head, Department of Chemistry, University of Kashmir, for providing the necessary facilities to carry out this work and thank the Central Instrumental Facility (CIF) department, JMI, Delhi and SAIF NEHU Shilong for providing XRD and TEM facilities, respectively. We thank Scientific and Industrial Research (CSIR), New Delhi for their financial assistance in the form of a junior research fellowship (JRF).

\section{References}

1 B. J. Kim, K. M. Bae, K. H. An and S. J. Park, Bull. Korean Chem. Soc., 2011, 32, 1321-1326.

2 N. J. Langford and R. E. Ferner, J. Hum. Hypertens., 1999, 13, 651-656.

3 T. Wajima and K. Sugawara, Fuel Process. Technol., 2011, 92, 1322-1327.

4 A. Bashir, S. Ahad and A. H. Pandith, Ind. Eng. Chem. Res., 2016, 55, 4820-4829.
5 G. Flora, D. Gupta and A. Tiwari, Interdiscip. Toxicol., 2012, 5, 47-58.

6 M. Dinari, R. Soltani and G. Mohammadnezhad, J. Chem. Eng. Data, 2017, 62, 2316-2329.

7 A. S. Adeleye and A. A. Keller, Water Res., 2014, 49, 236-250.

8 E. Hu, X. Gao, A. Etogo, Y. Xie, Y. Zhong and Y. Hu, J. Alloys Compd., 2014, 611, 335-340.

9 S. Wang, J. Li, X. Zhou, C. Zheng, J. Ning, Y. Zhonga and Y. Hu, J. Mater. Chem. A, 2014, 2, 19815-19821.

10 D. R. Tonini, D. A. Gauvin, R. W. Soffel and W. P. Freeman, Environ. Prog., 2003, 22, 167-173.

11 X. H. Wang, W. Y. Deng, Y. Y. Xie and C. Y. Wang, Chem. Eng. J., 2013, 228, 232-242.

12 G. Blanchard, M. Maunaye and G. Martin, Water Res., 1984, 18, 1501-1507.

13 K. G. Varshney, A. H. Pandith and U. Gupta, Langmuir, 1998, 14, 7353-7358.

14 K. G. Varshney and A. H. Pandith, Langmuir, 1999, 15, 74227425.

15 K. G. Varshney and A. H. Pandith, J. Indian Chem. Soc., 2001, 78, 250-253.

16 J. S. Liu, Y. Ma, T. W. Xu and G. Q. Shao, J. Hazard. Mater., 2010, 178, 1021-1029.

17 A. B. Albadarin, A. H. Al-Muhtaseb, N. A. Al-laqtah, G. M. Walker, S. J. Allen and M. N. M. Ahmad, Chem. Eng. J., 2011, 169, 20-30.

18 L. Ma, Q. Wang, S. M. Islam, Y. Liu, S. Ma and M. G. Kanatzidis, J. Am. Chem. Soc., 2016, 138, 2858-2866.

19 V. Tharanitharan and K. Srinivasan, Asian J. Chem., 2010, 22, 3036-3046.

20 H. Qian, Y. Hu, Y. Liu, M. Zhou and C. Guo, Mater. Lett., 2012, 68, 174-177.

21 A. Ali, H. Zafar, M. Zia, I. u. Haq, A. R. Phull, J. S. Ali and A. Hussain, Nanotechnol., Sci. Appl., 2016, 9, 49-67.

22 S. Chaudhary, A. Umar, K. K. Bhasin and S. Baskoutas, Materials, 2018, 11, 287, DOI: 10.3390/ma11020287.

23 L. Giraldo, A. Erto and J. C. Moreno-Piraján, Adsorption, 2012, 19, 465-474.

24 H. S. Hassan, M. F. Elkady, E. E. Hafez and E. Salama, Nanosci. Nanotechnol.-Asia, 2017, 7, 62-72.

25 X. H. Zhou, B. C. Huang, T. Zhou, Y. C. Liu and H. C. Shi, Chemosphere, 2015, 119, 568-576.

26 S. A. Elfeky, S. E. Mahmoud and A. F. Youssef, J. Adv. Res., 2017, 8, 435-443.

27 M. O. Ojemaye, O. O. Okoh and A. I. Okoh, Mater. Express, 2017, 7, 439-456.

28 A. Becker and K. Soliman, Neurochem. Res., 2009, 34, 16771684.

29 S. Lan, L. Liu, R. Li, Z. Leng and S. Gan, Ind. Eng. Chem. Res., 2014, 53, 3131-3139.

30 M. Fuji, Y. S. Han and C. Takai, KONA Powder Part. J., 2013, 30, 47-68.

31 X. Gao, H. B. Wu, L. Zheng, Y. Zhong, Y. Hu and X. W. Lou, Angew. Chem., Int. Ed., 2014, 53, 5917-5921.

32 Y. Wu, E. Hu, W. Dai, Z. Li, Y. Zhong and Y. Hu, RSC Adv., 2017, 7, 5093-5100. 
33 L. Mao, J. Li, Y. Xie, Y. Zhong and Y. Hu, RSC Adv., 2014, 4, 29698-29701.

34 M. B. Gawande, S. N. Shelke, R. Zboril and R. S. Varma, Acc. Chem. Res., 2014, 47, 1338-1348.

35 Y. Cho, C. H. Shin and S. Han, Nanoscale Res. Lett., 2016, 11, 1-9.

36 P. Pfaffli, A. Zitting and H. Vainio, Scand. J. Work, Environ. Health, 1978, 4, 22-27.

37 H. Noei, H. Qiu, Y. Wang, E. Loffler, C. Woll and M. Muhler, Phys. Chem. Chem. Phys., 2008, 10, 7092-7097.

38 A. Kumar, S. Arora, N. Mogha, S. S. Al-Deyab, Z. A. Ansari and S. G. Ansari, Energy Environ. Focus, 2013, 2, 101-107.

39 P. P. Sharmila and N. J. Tharayil, IOP Conf. Ser.: Mater. Sci. Eng., 2015, 73, 012019.

40 H. Kumar and R. Rani, Int. Lett. Chem., Phys. Astron., 2013, 14, 26-36.

41 A. H. Moharram, S. A. Mansour, M. A. Hussein and M. Rashad, J. Nanomater., 2014, 2014, 1-5.

42 S. Liufu, H. Xiao and Y. Li, Polym. Degrad. Stab., 2005, 87, 103-110.

43 V. T. Bhugul and G. N. Choudhari, International Journal of Scientific and Research Publications, 2015, 5(1), 2250-3153.

44 A. Marraa, C. Silvestrea, D. Duraccio and S. Cimminoa, Int. J. Biol. Macromol., 2016, 88, 254-262.

45 J. M. Berg, A. Romoser, N. Banarjee, R. Zebda and C. M. Sayes, Nanotoxicology, 2009, 3, 276-283.

46 D. T. Tran, RSC Adv., 2016, 6, 1339-1345.
47 S. Ahad, A. Bashir, T. Manzoor and A. H. Pandith, RSC Adv., 2016, 6, 35914-35927.

48 A. Stafiej and K. Pyrzynska, Sep. Purif. Technol., 2007, 58, 4952.

49 I. Langmuir, J. Am. Chem. Soc., 1918, 40, 1361-1403.

50 F. K. Onwu, C. U. Sonde and J. C. Igwe, J. Phys. Chem., 2014, 3, 89-95.

51 A. Farrukh, A. Akram, A. Ghaffar, S. Hanif, A. Hamid, H. Duran and B. Yameen, ACS Appl. Mater. Interfaces, 2013, 5, 3784-3793.

52 M. Monier, Int. J. Biol. Macromol., 2012, 50, 773-781.

53 J. J. Alcaraz-Espinoza, A. E. Chávez-Guajardo, J. C. MedinaLlamas, C. A. S. Andrade and C. P. de Melo, ACS Appl. Mater. Interfaces, 2015, 7, 7231-7240.

$54 \mathrm{H}$. Nakayama, S. Hirami and M. Tsuhako, J. Colloid Interface Sci., 2007, 315, 177-183.

55 Y. Zhao, Y. Chen, M. Li, S. Zhou, A. Xue and W. Xing, J. Hazard. Mater., 2009, 71, 640-646.

56 Z. H. Fard, C. D. Malliakas, J. L. Mertz and M. G. Kanatzidis, Chem. Mater., 2015, 27, 1925-1928.

57 M. J. Manos and M. G. Kanatzidis, Chem. - Eur. J., 2009, 15, 4779-4784.

58 M. Naushad, Z. A. ALOthman, M. R. Awual, M. M. Alam and G. E. Eldesoky, Ionics, 2015, 21, 2237-2245.

59 T. T. Liu, M. Yang, T. X. Wang and Q. P. Yuan, Ind. Eng. Chem. Res., 2012, 51, 454-463.

60 A. K. Bhattacharya, T. K. Naiya, S. N. Mandal and S. K. Das, Chem. Eng. J., 2008, 137, 529-541. 